\title{
Characteristics of adrenal insufficiency in hemodialysis patients
}

\author{
Naro Ohashi ${ }^{*}$, Yukitoshi Sakao ${ }^{1,2}$, Taichi Sato ${ }^{1}$, Sayaka Ishigaki ${ }^{3}$, Shinsuke Isobe ${ }^{1}$, Tomoyuki Fujikura',
} Akihiko Kato $^{3}$ and Hideo Yasuda ${ }^{1}$

\begin{abstract}
Background: Adrenal insufficiency causes abnormal subjective symptoms such as general fatigue, signs such as hypotension, and abnormalities such as hypoglycemia and leads to poor prognosis. However, all these are also observed in hemodialysis (HD) patients without adrenal insufficiency. The morphology of the adrenal glands in HD patients with adrenal insufficiency is unclear. Therefore, this study was performed to clarify the characteristics of adrenal insufficiency in HD patients.

Methods: Seventeen HD patients who had abnormal subjective symptoms and test results indicating adrenal insufficiency and whose serum cortisol levels were less than $18 \mu \mathrm{g} / \mathrm{dL}$ were recruited.

Results: Seven HD patients were diagnosed with adrenal insufficiency. No significant differences were found about abnormal subjective symptoms and images between patients with and without adrenal insufficiency. The levels of serum cortisol and serum cholinesterase were significantly lower in patients with adrenal insufficiency than in those without adrenal insufficiency. A plasma cortisol level of $8.45 \mu \mathrm{g} / \mathrm{dL}$ showed the highest sensitivity and specificity in the receiver operating characteristic curve. The serum cortisol levels were significantly and negatively associated with the plasma ferritin levels in patients with adrenal insufficiency. Multiple linear regression analyses revealed that the serum cortisol levels showed a significant negative association with the plasma ferritin levels after adjustments.

Conclusions: It is difficult to infer adrenal insufficiency in HD patients by subjective symptoms and images of the adrenal glands. Adrenal insufficiency correlates with nutritional and inflammatory status, and the levels of serum cholinesterase and plasma ferritin might reflect their corresponding status.
\end{abstract}

Keywords: Adrenal insufficiency, Cholinesterase, Cortisol, Ferritin, Hemodialysis

\section{Background}

Adrenal insufficiency is a life-threatening condition that occurs secondary to impaired secretion of adrenal glucocorticoid and mineralocorticoid hormones. This condition can be caused by primary destruction or dysfunction of the adrenal glands or impairment of the hypothalamic-pituitary-adrenal axis [1,2]. Adrenal insufficiency causes abnormal symptoms such as general

\footnotetext{
* Correspondence: ohashi-n@hama-med.ac.jp

${ }^{1}$ Internal Medicine 1, Hamamatsu University School of Medicine, 1-20-1 Handayama Higashi-ku, Hamamatsu 431-3192, Japan

Full list of author information is available at the end of the article
}

fatigue and appetite loss, abnormal signs such as hypotension, and abnormal laboratory findings such as hypoglycemia, eosinophilia, and electrolyte disorders such as hypercalcemia [1, 2]. Although adrenal insufficiency has been described in hemodialysis (HD) patients, reports are rare and diverse [3, 4]. In addition, HD patients frequently experience abnormal subjective symptoms, signs, and laboratory test results similar to adrenal insufficiency.

Barton et al. reported that dialysis-related amyloid depositions were found in 26 out of 30 adrenal glands in an autopsy study of $15 \mathrm{HD}$ patients [5]. However,

(C) The Author(s). 2021 Open Access This article is licensed under a Creative Commons Attribution 4.0 International License, which permits use, sharing, adaptation, distribution and reproduction in any medium or format, as long as you give appropriate credit to the original author(s) and the source, provide a link to the Creative Commons licence, and indicate if changes were made. The images or other third party material in this article are included in the article's Creative Commons licence, unless indicated otherwise in a credit line to the material. If material is not included in the article's Creative Commons licence and your intended use is not permitted by statutory regulation or exceeds the permitted use, you will need to obtain permission directly from the copyright holder. To view a copy of this licence, visit http://creativecommons.org/licenses/by/4.0/ The Creative Commons Public Domain Dedication waiver (http://creativecommons.org/publicdomain/zero/1.0/) applies to the data made available in this article, unless otherwise stated in a credit line to the data. 
Mazanec et al. reported that a patient receiving HD for 15 years developed systemic amyloidosis of $ß 2$ microglobulin. In the report, although noticeable depositions of amyloid were present in the myocardium, intervertebral discs, joint cartilages, and tendon, less conspicuous amounts were present in the blood vessel walls in the adrenal glands [6]. In this way, it is not known whether dialysis patients tend to have amyloid deposition in the adrenal gland and that amyloid deposition causes adrenal insufficiency.

As mentioned above, the significant symptoms, signs, and laboratory test data regarding adrenal insufficiency, causes of adrenal insufficiency, and morphology of adrenal glands have not been clarified in HD patients. Therefore, this study was performed to clarify the characteristics of adrenal insufficiency in HD patients.

\section{Methods}

\section{Patients}

We recruited 100 patients out of 185 patients undergoing regular $\mathrm{HD}$ at a single dialysis center (Hamana Clinic, Hamamatsu, Japan) who had agreed to participate in clinical research and were originally enrolled in our previous study (No. 23-185). We included the patients showing adrenal insufficiency with symptoms such as general fatigue and appetite loss; signs such as hypotension that was defined as systolic blood pressure (BP) $<100 \mathrm{mmHg}$ and/or stagger irrespective of the day with or without HD; and laboratory test results such as hypoglycemia that was defined as causal blood glucose levels $<70 \mathrm{mg} / \mathrm{dL}$, eosinophilia that was defined as eosinophil counts $>500 / \mu \mathrm{L}$, C-reactive protein (CRP) elevation that was defined as $>0.3 \mathrm{mg} / \mathrm{dL}$, and hypercalcemia that was defined as corrected calcium levels $>10.5 \mathrm{mg} / \mathrm{dL}$ among $100 \mathrm{HD}$ patients. Twentynine patients did not show symptoms, signs, or laboratory test results suspicious of adrenal insufficiency. On the other hand, we decided to exclude the patients who were being treated with steroids, as steroids are known to influence the hypothalamic-pituitary-adrenal axis, and who had significant clinical illness (e.g., cancer) among 100 patients. However, no patients fulfilled the exclusion criteria. Therefore, we examined the basal levels of serum cortisol in 71 patients. Blood samples were drawn in the morning fasting on non-dialysis day. Three patients whose serum cortisol levels were $\geq 18 \mu \mathrm{g} / \mathrm{dL}$ were excluded from this study because the possibility of adrenal insufficiency is ruled out when serum cortisol levels in the early morning are $\geq 18 \mu \mathrm{g} / \mathrm{dL}$ [7]. Because abnormal subjective symptoms, signs, and laboratory test results were not severe and this study needed 4-day, three-night hospitalization to perform close examination, most patients refused to participate in the clinical study. Finally, only 17 patients permitted to participate in this clinical study among 68 patients. The ethics committee of Hamamatsu University School of Medicine approved this study (No. 16-226), and we adhered to the principles of the Declaration of Helsinki. Written informed consent was obtained from all patients.

\section{Study protocols}

We recruited 17 patients with doubtful indications of adrenal insufficiency (symptoms, signs, and laboratory tests including serum cortisol levels). The patients were admitted to our hospital after undergoing a 2-day HD session at Hamana clinic. The following morning, corticotropin-releasing hormone $(\mathrm{CRH})$ stimulation tests were performed. Two days after admission, adrenocorticotropic hormone (ACTH) stimulation tests and chest radiography were performed before the start of the 1day HD session. Blood was drawn for laboratory tests before the start of the stimulation test. After HD session, bioimpedance analysis (BIA) of the body composition was performed under resting conditions (InBody Japan, Tokyo, Japan), and abdominal images were taken by computed tomography. BIA is a simple and non-invasive test measuring how low-voltage electric currents circulate through the body with the help of electrodes. Because BIA helps to distinguish how body fat, muscles, and body water are distributed in body tissues, it is widely used to determine patients' body composition [8]. Both the hormone stimulation tests were performed in the morning under resting conditions after overnight fasting. The patients' serum cortisol and plasma ACTH levels were measured by electrochemiluminescence immunoassays. Three days after admission, the patients were discharged.

\section{CRH and ACTH stimulation tests}

For CRH stimulation tests, after collecting basal level blood samples, we collected blood samples at 15, 30, 60, 90, and $120 \mathrm{~min}$ after intravenous injection of human $\mathrm{CRH}(100 \mu \mathrm{g})$ at bolus. ACTH stimulation tests were also performed in a manner similar to the $\mathrm{CRH}$ stimulation tests. After collecting basal level blood samples, we collected blood samples at 30 and $60 \mathrm{~min}$ after intravenous injection of synthetic ACTH $(250 \mu \mathrm{g})$ at bolus.

\section{Clinical data}

Serum cortisol levels were collected after overnight fasting and before admission at Hamana clinic, when the physicians suspected adrenal insufficiency. Dialysis dose (Kt/V urea) was assessed by the single-pooled urea kinetic model and the normalized protein catabolic rate (nPCR) as estimated protein intake was calculated using Shinzato's formula at Hamana clinic [9]. The patients' clinical data, such as age, sex, height, dry weight, and HD vintage, were recorded at the time of admission. 
Body mass index (BMI) was calculated by dividing body weight $(\mathrm{kg})$ by squared body height $(\mathrm{m})$. The patients' systolic and diastolic BPs were measured in the resting supine position before the start of the 1-day HD session.

Concentrations of some clinical parameters were measured in the clinical laboratory of the Hamamatsu University School of Medicine, University Hospital.

We corrected serum calcium levels with the following formula: corrected calcium $=$ measured calcium $+(4-$ serum albumin).

The percentages of muscle mass and body fat were calculated by muscle mass or body fat that was measured by BIA as follows: percentages of muscle mass or body fat $=$ the weight of muscle mass or body fat $/$ dry weight $\times 100$.

\section{Statistical analyses}

Results were expressed as the mean \pm standard deviation. Nonparametric data were expressed as median (interquartile range). The significance of differences among the diseases was determined using the analysis of variance (ANOVA) or Mann-Whitney $U$ test according to the distribution. The chi-square test was used to compare categorical variables.

The receiver operating characteristic (ROC) curves of the serum cortisol levels were examined to predict adrenal insufficiency.

Correlations between serum cortisol levels and other clinical parameters were evaluated using Pearson's product-moment correlation test. Multiple linear regression analyses were conducted to evaluate the relationships between serum cortisol levels and plasma ferritin levels. Age and sex were selected as independent variables because these parameters are common in performing multiple linear regression analyses.

We considered a $p$-value $<0.05$ to be statistically significant. Statistical analyses were performed with the IBM SPSS software, version 25 (IBM Corporation, Armonk, NY, USA).

\section{Results}

\section{Patient characteristics}

The characteristics of 68 patients suspected to have adrenal insufficiency are shown in Supplementary 1. The symptoms, signs, and laboratory tests indicating adrenal insufficiency were as follows: general fatigue, 9; appetite loss, 8; hypotension, 42; eosinophilia, 8; hypoglycemia, 4; hypercalcemia, 3; and CRP elevation, 25. Serum cortisol levels were $11.18 \pm 3.40 \mu \mathrm{g} / \mathrm{dL}$.

We recruited 17 patients to examine the presence or absence of adrenal insufficiency. The baseline characteristics of patients are shown in Table 1. The patients' age was $69.3 \pm 10.8$ years. The symptoms, signs, and laboratory tests indicating adrenal insufficiency were as follows: general fatigue, 4; appetite loss, 1; hypotension, 9; eosinophilia, 4; hypoglycemia, 0; hypercalcemia, 2; and CRP elevation, 5 . Serum cortisol levels were $9.84 \pm 3.78$ $\mu \mathrm{g} / \mathrm{dL}$.

\section{Diagnoses of adrenal insufficiency by CRH and ACTH stimulation tests in all patients}

Table 2 shows results of the diagnoses of adrenal insufficiency by $\mathrm{CRH}$ and $\mathrm{ACTH}$ stimulation tests in all patients. At first, we diagnosed patients with adrenal insufficiency when the peak plasma ACTH or serum cortisol levels were less than two-fold higher than their basal levels and/or when their peak serum cortisol levels were less than $18 \mu \mathrm{g} / \mathrm{dL}$, as described previously (diagnosis criteria A) [4]. Although the diagnostic criteria for adrenal insufficiency have not been completely determined, some specialist opinions have been expressed in this field [7]. Therefore, we have diagnosed adrenal insufficiency and classified it into 3 categories as follows: diseases of the adrenal gland (primary), interference with $\mathrm{ACTH}$ secretion by the pituitary gland (secondary), or interference with $\mathrm{CRH}$ secretion by the hypothalamus (tertiary) [10]. The following are specialists' opinions: (1) When the peak serum cortisol level is $<15 \mu \mathrm{g} / \mathrm{dL}$ in the ACTH stimulation test, primary adrenal insufficiency should be suspected (diagnosis criteria B) (2). When the basal plasma ACTH levels are high without increased serum cortisol levels in the $\mathrm{CRH}$ and/or ACTH stimulation tests, primary adrenal insufficiency should be suspected (diagnosis criteria C). (3) When the basal plasma ACTH level is low or normal and response to ACTH is low or absent in the CRH stimulation test, secondary adrenal insufficiency should be suspected (diagnosis criteria $\mathrm{D}$ ). After referring to some specialist opinions, the tentative diagnoses were finally made as a result of discussion with some physicians, including nephrologists and endocrinologists who specialized in adrenal insufficiency. Seven HD patients were tentatively diagnosed as having adrenal insufficiency. Five and two patients were tentatively diagnosed as having primary and secondary adrenal insufficiencies, respectively.

\section{Comparison of incidence rate (\%) of abnormal subjective symptoms, signs, laboratory test results, and images of the adrenal gland between patients with and without adrenal insufficiency}

We compared the incidence rate (\%) of abnormal subjective symptoms, signs, and laboratory test results between patients with and without adrenal insufficiency (Table 3).

Although eosinophilia tended to be detected in the patients with adrenal insufficiency more frequently than those without adrenal insufficiency, there were no significant differences. Moreover, no significant differences 
Table 1 Patient characteristics that were admitted to examine the adrenal insufficiency

\begin{tabular}{|c|c|}
\hline Age (year) & $69.3 \pm 10.8$ \\
\hline Sex & Male 11/female 6 \\
\hline Causes of ESRD & DKD 2/CGN 11/NS 1/other 3 \\
\hline Hemodialysis vintage (months) & 175 [120-396] \\
\hline Previous history of steroid usage & Existence 2/non-existence 15 \\
\hline $\begin{array}{l}\text { Symptoms, signs, and laboratory tests indicating } \\
\text { adrenal insufficiency }\end{array}$ & $\begin{array}{l}\text { General fatigue 4, appetite loss 1, hypotension 9, eosinophilia 4, hypoglycemia 0, } \\
\text { hypercalcemia 2, CRP elevation } 5\end{array}$ \\
\hline Height (cm) & $159.5 \pm 8.5$ \\
\hline Dry weight (kg) & $52.0 \pm 10.8$ \\
\hline Body mass index $\left(\mathrm{kg} / \mathrm{m}^{2}\right)$ & $20.3 \pm 2.9$ \\
\hline Blood pressure (mmHg) & $142.2 \pm 24.8 / 76.6 \pm 15.1$ \\
\hline Muscle mass (\%) & $14.0 \pm 1.5$ \\
\hline Body fat (\%) & $27.3 \pm 7.3$ \\
\hline Cardiothoracic ratio (\%) & $52.6 \pm 4.2$ \\
\hline $\mathrm{Kt} / \mathrm{N}$ urea & $1.56 \pm 0.26$ \\
\hline nPCR (g/kg/day) & $0.94 \pm 0.14$ \\
\hline 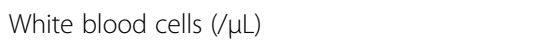 & $5027.7 \pm 1732.6$ \\
\hline Eosinophil counts $(/ \mu \mathrm{L})$ & $225.5 \pm 155.2$ \\
\hline Lymphocyte counts $(/ \mu \mathrm{L})$ & $977.0 \pm 338.7$ \\
\hline Hemoglobin (g/dL) & $10.68 \pm 1.37$ \\
\hline Sodium (mEq/L) & $138.2 \pm 3.3$ \\
\hline Potassium (mEq/L) & $4.41 \pm 0.78$ \\
\hline Chloride (mEq/L) & $101.9 \pm 3.1$ \\
\hline Corrected calcium (mg/dL) & $9.32 \pm 0.86$ \\
\hline Phosphate (mg/dL) & $4.30 \pm 1.70$ \\
\hline Blood urea nitrogen (mg/dL) & $47.1 \pm 15.0$ \\
\hline Creatinine $(\mathrm{mg} / \mathrm{dL})$ & $9.28 \pm 2.18$ \\
\hline Albumin (g/dL) & $3.32 \pm 0.56$ \\
\hline Prealbumin (mg/dL) & $25.40 \pm 6.37$ \\
\hline Cholinesterase (U/L) & $239.3 \pm 84.1$ \\
\hline Casual blood glucose (mg/dL) & $98.2 \pm 22.2$ \\
\hline Ferrum $(\mu \mathrm{g} / \mathrm{dL})$ & $79.2 \pm 21.4$ \\
\hline $\operatorname{TIBC}(\mu \mathrm{g} / \mathrm{dL})$ & $244.5 \pm 51.1$ \\
\hline Ferritin $(\mathrm{ng} / \mathrm{mL})$ & $131.3 \pm 191.2$ \\
\hline $\operatorname{CRP}(\mathrm{mg} / \mathrm{dL})$ & $0.43 \pm 0.59$ \\
\hline ß2-microglobulin (mg/L) & $25.40 \pm 6.37$ \\
\hline Cortisol ( $\mu \mathrm{g} / \mathrm{dL})$ & $9.84 \pm 3.78$ \\
\hline i-PTH (pg/mL) & $172.1 \pm 130.7$ \\
\hline
\end{tabular}

Abbreviations: ESRD end-stage renal disease, DKD diabetic kidney disease, CGN chronic glomerulonephritis, NS nephrosclerosis, $n P C R$ normalized protein catabolic rate, TIBC total iron-binding capacity, CRP C-reactive protein, i-PTH intact-parathyroid hormone

were found with regard to adrenal insufficiencyrepresentative subjective symptoms (general fatigue and appetite loss), signs (hypotension), and laboratory test results (hypercalcemia and CRP elevation) between patients with and without adrenal insufficiency. There were no patients with hypoglycemia in both groups. In addition, we compared the incidence rate (\%) of abnormal images of the adrenal gland between patients with and without adrenal insufficiency. Atrophic adrenal gland was found in one patient $(14.3 \%)$ with adrenal insufficiency; adrenal adenoma and enlargement of the adrenal gland were found in one $(10.0 \%)$ and two patients 
Table 2 Diagnoses of adrenal insufficiency (Al) by corticotropin-releasing hormone (CRH) and adrenocorticotropic hormone (ACTH) stimulation tests

\begin{tabular}{|c|c|c|c|c|c|c|c|c|c|c|}
\hline & & & 0 & 15 & 30 & 60 & 90 & 120 (min) & Diagnosis criteria & Diagnosis \\
\hline \multirow[t]{3}{*}{1} & CRH test & ACTH & 24.4 & 67.0 & 90.0 & 93.4 & 47.0 & 48.0 & $A$ & Normal response \\
\hline & & Cortisol & 6.1 & 10.2 & 13.9 & 15.0 & 14.5 & 11.9 & & \\
\hline & ACTH test & Cortisol & 4.2 & & 15.6 & 18.8 & & & & \\
\hline \multirow[t]{3}{*}{2} & CRH test & ACTH & 179.9 & 265.8 & 231.6 & 257.8 & 234.5 & 209.7 & C & Primary Al \\
\hline & & Cortisol & 4.3 & 4.7 & 5.0 & 5.2 & 5.0 & 4.9 & & \\
\hline & ACTH test & Cortisol & 5.7 & & 7.7 & 8.1 & & & & \\
\hline \multirow[t]{3}{*}{3} & CRH test & ACTH & 73.3 & 136.2 & 140.5 & 127.5 & 95.1 & 71.8 & C & Primary Al \\
\hline & & Cortisol & 6.1 & 8.3 & 10.0 & 10.5 & 8.7 & 7.1 & & \\
\hline & ACTH test & Cortisol & 6.0 & & 11.1 & 13.5 & & & & \\
\hline \multirow[t]{3}{*}{4} & CRH test & ACTH & 18.3 & 42.1 & 50.8 & 55.1 & 37.5 & 33.9 & B & Normal response \\
\hline & & Cortisol & 8.1 & 10.7 & 12.2 & 13.3 & 12.0 & 11.0 & & \\
\hline & ACTH test & Cortisol & 9.4 & & 15.4 & 17.4 & & & & \\
\hline \multirow[t]{3}{*}{5} & CRH test & ACTH & 54.2 & 134.0 & 129.0 & 101.0 & 71.4 & 54.3 & B & Normal response \\
\hline & & Cortisol & 8.6 & 11.7 & 13.5 & 13.7 & 12.4 & 11.1 & & \\
\hline & ACTH test & Cortisol & 10.6 & & 15.9 & 17.3 & & & & \\
\hline \multirow[t]{3}{*}{6} & CRH test & ACTH & 33.2 & 98.9 & 100.0 & 104.0 & 69.1 & 46.4 & A & Normal response \\
\hline & & Cortisol & 10.2 & 11.7 & 14.3 & 16.5 & 16.6 & 15.8 & & \\
\hline & ACTH test & Cortisol & 12.0 & & 17.8 & 19.2 & & & & \\
\hline \multirow[t]{3}{*}{7} & CRH test & ACTH & 42.1 & 55.3 & 54.3 & 41.1 & 34.3 & 36.8 & $A, B$, and $D$ & Secondary Al \\
\hline & & Cortisol & 9.1 & 10.6 & 11.5 & 10.4 & 8.8 & 7.6 & & \\
\hline & ACTH test & Cortisol & 7.6 & & 15.0 & 16.7 & & & & \\
\hline \multirow[t]{3}{*}{8} & CRH test & ACTH & 10.8 & 33.4 & 32.7 & 26.1 & 17.7 & 14.6 & A & Normal response \\
\hline & & Cortisol & 9.8 & 15.0 & 19.1 & 19.9 & 16.8 & 14.2 & & \\
\hline & ACTH test & Cortisol & 7.8 & & 22.8 & 27.2 & & & & \\
\hline \multirow[t]{3}{*}{9} & CRH test & $\mathrm{ACTH}$ & 26.3 & 53.4 & 53.9 & 37.4 & 29.9 & 20.7 & A & Normal response \\
\hline & & Cortisol & 9.9 & 13.2 & 15.1 & 15.0 & 13.1 & 11.7 & & \\
\hline & ACTH test & Cortisol & 12.0 & & 19.8 & 22.9 & & & & \\
\hline \multirow[t]{3}{*}{10} & CRH test & ACTH & 32.1 & 43.2 & 49.9 & 41.9 & 29.1 & 25.6 & A & Normal response \\
\hline & & Cortisol & 13.2 & 15.1 & 16.4 & 16.9 & 13.7 & 11.7 & & \\
\hline & ACTH test & Cortisol & 10.9 & & 20.6 & 22.9 & & & & \\
\hline \multirow[t]{3}{*}{11} & CRH test & ACTH & 26.0 & 34.5 & 42.9 & 41.9 & 48.9 & 40.6 & A & Normal response \\
\hline & & Cortisol & 14.9 & 14.8 & 16.8 & 17.1 & 17.7 & 16.4 & & \\
\hline & ACTH test & Cortisol & 13.2 & & 22.6 & 24.5 & & & & \\
\hline \multirow[t]{3}{*}{12} & CRH test & ACTH & 96.5 & 120.0 & 131.0 & 111.0 & 97.5 & 72.0 & C & Primary Al \\
\hline & & Cortisol & 5.6 & 6.9 & 7.5 & 6.6 & 5.2 & 4.5 & & \\
\hline & ACTH test & Cortisol & 5.3 & & 9.4 & 10.3 & & & & \\
\hline \multirow[t]{3}{*}{13} & CRH test & ACTH & 13.7 & 52.3 & 73.7 & 64.9 & 56.2 & 69.9 & A & Normal response \\
\hline & & Cortisol & 12.6 & 14.2 & 18.3 & 21.2 & 22.2 & 23.6 & & \\
\hline & ACTH test & Cortisol & 13.0 & & 27.8 & 33.4 & & & & \\
\hline \multirow[t]{3}{*}{14} & CRH test & ACTH & 44.2 & 117.0 & 106.0 & 74.8 & 49.5 & 32.1 & A & Normal response \\
\hline & & Cortisol & 10.3 & 12.7 & 13.9 & 13.5 & 11.6 & 9.5 & & \\
\hline & ACTH test & Cortisol & 6.5 & & 14.9 & 18.2 & & & & \\
\hline 15 & CRH test & ACTH & 114.1 & 158.4 & 150.8 & 124.8 & 102.6 & 87.6 & C & Primary Al \\
\hline
\end{tabular}


Table 2 Diagnoses of adrenal insufficiency (AI) by corticotropin-releasing hormone (CRH) and adrenocorticotropic hormone (ACTH) stimulation tests (Continued)

\begin{tabular}{|c|c|c|c|c|c|c|c|c|c|c|}
\hline & & & 0 & 15 & 30 & 60 & 90 & 120 (min) & Diagnosis criteria & Diagnosis \\
\hline & & Cortisol & 9.3 & 9.8 & 9.9 & 9.8 & 9.5 & 9.2 & & \\
\hline & ACTH test & Cortisol & 15.9 & & 17.1 & 17.4 & & & & \\
\hline \multirow[t]{3}{*}{16} & CRH test & ACTH & 37.2 & 41.6 & 35.4 & 40.1 & 42.2 & 35.1 & $A$ and $B$ & Primary Al \\
\hline & & Cortisol & 12.6 & 12.8 & 12.3 & 12.4 & 12.6 & 12.1 & & \\
\hline & ACTH test & Cortisol & 3.7 & & 9.6 & 11.8 & & & & \\
\hline \multirow[t]{3}{*}{17} & CRH test & ACTH & 33.5 & 41.1 & 41.3 & 43.4 & 35.7 & 35.8 & $A, B$, and D & Secondary Al \\
\hline & & Cortisol & 9.1 & 8.7 & 9.4 & 9.1 & 8.3 & 7.5 & & \\
\hline & ACTH test & Cortisol & 7.5 & & 15.0 & 14.7 & & & & \\
\hline
\end{tabular}

(20.0\%) without adrenal insufficiency, respectively (data not shown).

\section{Comparison of each parameter in patients with and without adrenal insufficiency}

We compared each parameter in patients with and without adrenal insufficiency (Table 4). No significant differences were found regarding previous history of steroid usage and present erythropoiesis-stimulating agent (ESA) usage between the patients with and without adrenal insufficiency. Levels of serum cortisol were significantly lower in patients with adrenal insufficiency than those without adrenal insufficiency (serum cortisol 7.66 $\pm 2.35 \mu \mathrm{g} / \mathrm{dL}$ vs. $11.38 \pm 3.92 \mu \mathrm{g} / \mathrm{dL} ; p=0.041)$. Moreover, levels of serum cholinesterase were significantly lower in patients with adrenal insufficiency than those without adrenal insufficiency (serum cholinesterase $177.4 \pm 72.1 \mathrm{U} / \mathrm{L}$ vs. $282.6 \pm 63.7 \mathrm{U} / \mathrm{L} ; p<0.01)$. Meanwhile, eosinophil counts and plasma ferritin levels tended to be higher in patients with adrenal insufficiency than in those without adrenal insufficiency (eosinophil counts $302.8 \pm 172.7 / \mu \mathrm{L}$ vs. $171.4 \pm 122.7 / \mu \mathrm{L} ; p=0.085$ and plasma ferritin $234.4 \pm 317.9 \mathrm{ng} / \mathrm{mL}$ vs. $79.8 \pm 55.7$ $\mathrm{ng} / \mathrm{mL} ; p=0.15)$. In addition, serum prealbumin levels tended to be lower in patients with adrenal insufficiency than in those without adrenal insufficiency (serum prealbumin $21.18 \pm 6.73 \mathrm{mg} / \mathrm{dL}$ vs. $27.09 \pm 5.70 \mathrm{mg} / \mathrm{dL} ; p=$
0.12). No significant differences were found in other parameters between patients with and without adrenal insufficiency.

\section{ROC curve of the serum cortisol levels}

The ROC curve of the serum cortisol levels was examined to predict adrenal insufficiency. The area under the ROC showed 0.81, and the serum cortisol level $8.45 \mu \mathrm{g} /$ $\mathrm{dL}$ indicated highest sensitivity $(80.0 \%)$ and specificity (71.4\%) in the ROC curve (Fig. 1).

\section{Relationships between serum cortisol levels and other clinical parameters in the patients with adrenal insufficiency}

We examined correlations between serum cortisol levels and other clinical parameters by Pearson's product-moment correlation test in the patients with adrenal insufficiency (Table 5). Serum cortisol levels were significantly and negatively associated with plasma ferritin levels $(r=-0.89, p=$ 0.018). By contrast, no significant relationships between serum cortisol levels and other parameters were found.

\section{Multiple linear regression analyses of serum cortisol levels and the clinical parameters including plasma ferritin levels in the patients with adrenal insufficiency Multiple linear regression analysis revealed that serum cortisol levels had a significant negative association with}

Table 3 Comparison of incidence rate (\%) between patients with and without adrenal insufficiency

\begin{tabular}{llll}
\hline & Patients with adrenal insufficiency & Patients without adrenal insufficiency & P-value \\
\hline General fatigue & $28.6 \%$ & $20.0 \%$ & 0.56 \\
Appetite loss & $14.3 \%$ & $0 \%$ & 0.41 \\
Hypotension & $57.1 \%$ & $50.0 \%$ & 0.36 \\
Eosinophilia & $42.9 \%$ & $10.0 \%$ & 0.15 \\
Hypoglycemia & $0 \%$ & $0 \%$ & $\mathrm{NA}$ \\
Hypercalcemia & $14.3 \%$ & $10.0 \%$ & 0.52 \\
CRP elevation & $42.9 \%$ & $20.0 \%$ & 0.26 \\
\hline
\end{tabular}

Abbreviation: NA not applicable, CRP C-reactive protein 
Table 4 Comparison of each parameter in patients with and without adrenal insufficiency

\begin{tabular}{|c|c|c|c|}
\hline & Patients with adrenal insufficiency & Patients without adrenal insufficiency & $P$-value \\
\hline Age (year) & $66.4 \pm 13.0$ & $71.3 \pm 9.0$ & 0.38 \\
\hline Sex & Male 6/female 1 & Male 5/female 5 & 0.13 \\
\hline Patients with and without previous history of steroid usage & User 1/non-user 6 & User $1 /$ non-user 9 & 0.67 \\
\hline Patients with and without present ESA usage & User 3/non-user 4 & User 8/non-user 2 & 0.12 \\
\hline HD vintage (months) & $285.1 \pm 118.6$ & $182.6 \pm 145.9$ & 0.15 \\
\hline Height $(\mathrm{cm})$ & $164.2 \pm 7.6$ & $156.2 \pm 7.9$ & 0.054 \\
\hline Dry weight (kg) & $55.7 \pm 8.6$ & $49.4 \pm 11.8$ & 0.24 \\
\hline Body mass index $\left(\mathrm{kg} / \mathrm{m}^{2}\right)$ & $20.59 \pm 2.07$ & $20.01 \pm 3.44$ & 0.70 \\
\hline Systolic BP (mmHg) & $143.6 \pm 22.1$ & $141.2 \pm 27.6$ & 0.85 \\
\hline Diastolic BP (mmHg) & $77.1 \pm 19.5$ & $76.2 \pm 12.3$ & 0.90 \\
\hline Muscle mass (\%) & $13.8 \pm 1.3$ & $14.1 \pm 1.6$ & 0.72 \\
\hline Body fat (\%) & $29.2 \pm 5.8$ & $26.5 \pm 7.9$ & 0.55 \\
\hline Cardiothoracic ratio (\%) & $52.8 \pm 3.6$ & $52.5 \pm 4.6$ & 0.90 \\
\hline $\mathrm{Kt} / \mathrm{N}$ urea & $1.59 \pm 0.18$ & $1.55 \pm 0.32$ & 0.77 \\
\hline nPCR (g/kg/day) & $0.91 \pm 0.10$ & $0.95 \pm 0.16$ & 0.59 \\
\hline White blood cells $(/ \mu \mathrm{L})$ & $4857.1 \pm 1790.1$ & $5147.0 \pm 1778.2$ & 0.75 \\
\hline Eosinophil counts $(/ \mu \mathrm{L})$ & $302.8 \pm 172.7$ & $171.4 \pm 122.7$ & 0.085 \\
\hline Lymphocyte counts (/ML) & $999.5 \pm 317.3$ & $961.3 \pm 369.0$ & 0.83 \\
\hline Hemoglobin $(\mathrm{g} / \mathrm{dL})$ & $10.57 \pm 1.67$ & $10.76 \pm 1.22$ & 0.79 \\
\hline Sodium (mEq/L) & $136.9 \pm 2.6$ & $139.2 \pm 3.4$ & 0.15 \\
\hline Potassium (mEq/L) & $3.93 \pm 0.88$ & $4.69 \pm 0.59$ & 0.056 \\
\hline Chloride (mEq/L) & $100.4 \pm 1.8$ & $102.9 \pm 3.5$ & 0.11 \\
\hline Corrected calcium (mg/dL) & $9.66 \pm 1.07$ & $9.08 \pm 0.62$ & 0.18 \\
\hline Phosphate (mg/dL) & $3.94 \pm 2.21$ & $4.55 \pm 1.31$ & 0.49 \\
\hline Blood urea nitrogen $(\mathrm{mg} / \mathrm{dL})$ & $41.6 \pm 17.2$ & $50.9 \pm 12.7$ & 0.21 \\
\hline Creatinine $(\mathrm{mg} / \mathrm{dL})$ & $8.89 \pm 2.85$ & $9.56 \pm 1.69$ & 0.55 \\
\hline Albumin (g/dL) & $3.10 \pm 0.75$ & $3.47 \pm 0.36$ & 0.19 \\
\hline Prealbumin (mg/dL) & $21.18 \pm 6.73$ & $27.09 \pm 5.70$ & 0.12 \\
\hline Cholinesterase (U/L) & $177.4 \pm 72.1$ & $282.6 \pm 63.7$ & $<0.01$ \\
\hline Casual blood glucose (mg/dL) & $98.1 \pm 7.8$ & $98.2 \pm 29.0$ & 1.00 \\
\hline Ferrum $(\mu \mathrm{g} / \mathrm{dL})$ & $88.2 \pm 16.3$ & $74.7 \pm 22.9$ & 0.26 \\
\hline $\mathrm{TIBC}(\mu \mathrm{g} / \mathrm{dL})$ & $235.6 \pm 62.7$ & $249.0 \pm 47.3$ & 0.65 \\
\hline Ferritin (ng/mL) & $234.4 \pm 317.9$ & $79.8 \pm 55.7$ & 0.15 \\
\hline CRP (mg/dL) & $0.51 \pm 0.67$ & $0.38 \pm 0.55$ & 0.66 \\
\hline ß2-microglobulin (mg/L) & $27.53 \pm 4.81$ & $24.34 \pm 4.71$ & 0.28 \\
\hline Cortisol ( $\mu \mathrm{g} / \mathrm{dL})$ & $7.66 \pm 2.35$ & $11.38 \pm 3.92$ & 0.041 \\
\hline i-PTH (pg/mL) & $158.6 \pm 116.9$ & $178.9 \pm 142.6$ & 0.79 \\
\hline
\end{tabular}

Abbreviations: ESA erythropoiesis-stimulating agent, $H D$ hemodialysis, $B P$ blood pressure, $n P C R$ normalized protein catabolic rate, $T I B C$ total iron binding capacity, CRP C-reactive protein, i-PTH intact-parathyroid hormone

plasma ferritin levels after adjustments for age and sex $(\beta=-0.97$ and $p<0.01)$ (data not shown).

\section{Discussion}

This study indicated that it is difficult to infer adrenal insufficiency in HD patients by using subjective symptoms, signs, and images of adrenal glands; and serum cortisol level of $8.45 \mu \mathrm{g} / \mathrm{dL}$ had the highest sensitivity and specificity to diagnose adrenal insufficiency in HD patients. In addition, this study indicated that adrenal insufficiency correlated with nutritional and inflammatory status and that levels of serum cholinesterase and plasma ferritin might be useful markers to predict adrenal insufficiency in HD patients.

Although HD may be one of the causes for adrenal insufficiency [3, 4], adrenal insufficiency in HD patients is rare. However, seven patients with adrenal insufficiency were found from the recruited 17 patients with 


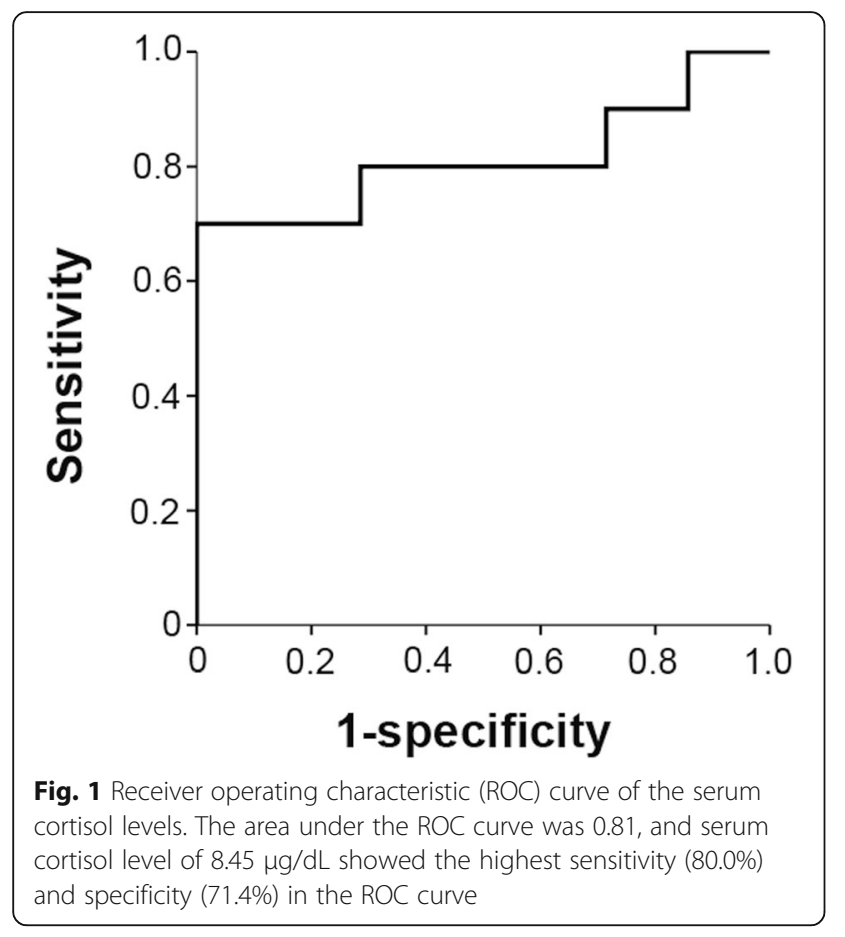

symptoms, signs, and laboratory tests indicating adrenal insufficiency and whose fasting serum cortisol levels were less than $18 \mu \mathrm{g} / \mathrm{dL}$. We suggest that physicians who have doubts of adrenal insufficiency measure serum cortisol levels and perform hormone stimulation tests to detect adrenal insufficiency.

Adrenal insufficiency causes several abnormal symptoms such as fatigue, signs such as hypotension and weight loss, and laboratory test results such as eosinophilia and hypoglycemia. However, HD patients frequently experience similar abnormal subjective symptoms, signs, and laboratory test results. Bossola et al. reported that 164 patients (60.5\%) suffered from post-dialysis fatigue in 271 chronic HD patients [11]. Kuipers et al. indicated that when intradialytic hypotension was defined as a decrease in systolic blood pressure $\geq 20 \mathrm{mmHg}$ or mean arterial pressure $\geq 10$ $\mathrm{mmHg}$, intradialytic hypotension occurred in $77.7 \%$ of the 3818 HD sessions [12]. Eosinophilia is primarily caused by contact of blood with the dialyzer in HD patients [13], and Kang et al. showed median eosinophil count of 231 cells $/ \mu \mathrm{L}$ and occurrence of eosinophilia $(>350$ cells $/ \mu \mathrm{L}$ ) in $23.4 \%$ of the $107,506 \mathrm{HD}$ patients [14]. Electrolyte disorders, such as hypercalcemia due to impaired urinary calcium excretion and increased bone reabsorption, are sometimes experienced by HD patients [4]. Moreover, aside from HD patients with diabetes mellitus, some clinical conditions, such as malnutrition, infection, and multiorgan failure, result in hypoglycemia in non-diabetic HD patients [15]. Therefore, it is difficult to infer adrenal insufficiency in $\mathrm{HD}$ patients by
Table 5 Relationships between serum cortisol levels and other clinical parameters in the patients with adrenal insufficiency

\begin{tabular}{|c|c|c|}
\hline & $r$ & $P$-value \\
\hline Age (year) & -0.16 & 0.73 \\
\hline Hemodialysis vintage (months) & -0.46 & 0.30 \\
\hline Height $(\mathrm{cm})$ & -0.50 & 0.25 \\
\hline Dry weight (kg) & 0.027 & 0.95 \\
\hline Body mass index $\left(\mathrm{kg} / \mathrm{m}^{2}\right)$ & 0.44 & 0.33 \\
\hline Systolic BP (mmHg) & 0.35 & 0.44 \\
\hline Diastolic BP (mmHg) & 0.038 & 0.94 \\
\hline Muscle mass (\%) & -0.41 & 0.59 \\
\hline Body fat (\%) & 0.48 & 0.52 \\
\hline Cardiothoracic ratio (\%) & 0.13 & 0.83 \\
\hline Kt/N urea & -0.31 & 0.23 \\
\hline $\mathrm{nPCR}$ (g/kg/day) & 0.22 & 0.39 \\
\hline White blood cells $(/ \mu \mathrm{L})$ & 0.59 & 0.16 \\
\hline Eosinophil counts $(/ \mu \mathrm{L})$ & -0.12 & 0.80 \\
\hline Lymphocyte counts $(/ \mu \mathrm{L})$ & 0.16 & 0.74 \\
\hline Hemoglobin (g/dL) & 0.47 & 0.29 \\
\hline Sodium (mEq/L) & 0.26 & 0.58 \\
\hline Potassium (mEq/L) & -0.11 & 0.83 \\
\hline Chloride (mEq/L) & 0.12 & 0.80 \\
\hline Corrected calcium (mg/dL) & 0.38 & 0.40 \\
\hline Phosphate (mg/dL) & 0.33 & 0.52 \\
\hline Blood urea nitrogen (mg/dL) & 0.16 & 0.73 \\
\hline Creatinine $(\mathrm{mg} / \mathrm{dL})$ & 0.32 & 0.48 \\
\hline Albumin (g/dL) & 0.15 & 0.74 \\
\hline Prealbumin (mg/dL) & -0.12 & 0.88 \\
\hline Cholinesterase (U/L) & 0.39 & 0.38 \\
\hline Casual blood glucose (mg/dL) & -0.017 & 0.97 \\
\hline Ferrum $(\mu \mathrm{g} / \mathrm{dL})$ & -0.44 & 0.39 \\
\hline $\mathrm{TIBC}(\mu \mathrm{g} / \mathrm{dL})$ & 0.39 & 0.45 \\
\hline Ferritin $(\mathrm{ng} / \mathrm{mL})$ & -0.89 & 0.018 \\
\hline CRP $(\mathrm{mg} / \mathrm{dL})$ & -0.044 & 0.93 \\
\hline ß2-microglobulin (mg/L) & -0.071 & 0.93 \\
\hline i-PTH (pg/mL) & -0.24 & 0.70 \\
\hline
\end{tabular}

Abbreviations: $n P C R$ normalized protein catabolic rate, $B P$ blood pressure, $T I B C$ total iron-binding capacity, CRP C-reactive protein, $i-P T H$ intact-parathyroid hormone

abnormal subjective symptoms, signs, and laboratory test results. No significant differences were found between HD patients with and without adrenal insufficiency in this study.

It is possible that adrenal insufficiency in dialysis patients is caused by deposition of amyloid protein in the adrenal gland [5]. Deposition of amyloid protein in some organs result in their swelling. In this study, whereas atrophic adrenal gland was found in one patient (14.3\%) 
with adrenal insufficiency, it is difficult to infer adrenal insufficiency in HD patients by images of the adrenal gland.

In this study, only serum cholinesterase levels were significantly different between the HD patients with and without adrenal insufficiency. Cortisol is a steroid hormone that binds to its receptor and interacts with the DNA-binding domain. Almost 1000-2000 genes are sensitive to their effects, including immune/inflammatory response genes [16]. Inflammation and malnutrition correlate with each other in HD patients, and the antiinflammatory effect leads to improvement of nutritional status [17]. In this study, neither muscle mass (\%) and body fat (\%) measured by BIA nor lymphocyte counts were significantly different between the HD patients with and without adrenal insufficiency. In addition, although a lower tendency of prealbumin levels was noted in the HD patients with adrenal insufficiency, there were no significant differences between HD patients with and without adrenal insufficiency. On the other hand, significant differences were found in serum cholinesterase levels between HD patients with and without adrenal insufficiency. Moreover, levels of serum cholinesterase were significantly and positively associated with BMI, blood urea nitrogen, serum creatinine, and serum albumin in HD patients with adrenal insufficiency in this study (data not shown). Serum cholinesterase is not only a marker for liver synthetic capacity but also a marker to represent nutritional status. Our previous study indicated that serum cholinesterase level increased first as the marker for improvement of nutrition status following eradication of Helicobacter pylori [18]. In addition, Yamamoto et al. reported that the combination of serum cholinesterase and albumin levels was useful for nutritional status to predict the prognosis of patients with colorectal cancer [19], and Sato et al. indicated that serum cholinesterase level was an important prognostic factor in chronic heart failure [20]. According to the contents mentioned above, adrenal insufficiency causes inflammatory response due to a relative deficiency of cortisol, and inflammation leads to the exacerbation of nutritional status. Therefore, serum cholinesterase, one of the excellent markers to reflect the nutritional status, may indicate the significant difference in the patients with and without adrenal insufficiency. The situation in HD patients coincides with the fact that the serum cholinesterase levels of patients with adrenal insufficiency are lower than those of patients without adrenal insufficiency, reflecting chronic malnutrition in the general population.

As mentioned above, cortisol is a steroid hormone, and it correlates with immune/inflammatory response genes [16]. A relative deficiency of cortisol exacerbates chronic inflammation. When chronic inflammation is induced, hepcidin, a protein synthesized in the liver, contributes to decreasing serum iron by reducing intestinal iron absorption and impairing iron release from macrophages. Therefore, levels of plasma ferritin, a storage iron, are increased by chronic inflammation [21]. As a result, plasma cortisol levels were significantly and negatively associated with plasma ferritin levels even after adjustment for age and sex in this study. In contrast, CRP is known to be a marker of inflammation, and it elevates in multiple stages of inflammation during the acute phase [22]. Therefore, we speculate that because CRP is influenced by several causes (infection, inflammation, and injury), including adrenal insufficiency, plasma cortisol levels associated with chronic inflammation were not associated with serum CRP levels in this study.

This study has some limitations. First, the sample size was relatively small. This study was performed in a single-center cohort, and some patients refused to participate in this study because the study demanded close examinations in the hospital. Therefore, it is possible that eosinophil counts and levels of serum prealbumin and plasma ferritin did not show significant differences between patients with and without adrenal insufficiency, although a tendency was observed with these parameters. It is necessary to confirm this study results by large-scale clinical research. Second, we were worried about accurately diagnosing adrenal insufficiency. After referring to some specialist opinions, the tentative diagnoses were finally made by discussion with some physicians, including nephrologists and endocrinologists who specialize in adrenal insufficiency. As a result, we could detect significant differences in serum cortisol levels between the HD patients with and without adrenal insufficiency. Because steroid supplementation is not mandatory to diagnose adrenal insufficiency and the symptoms, signs, and laboratory test results indicating adrenal insufficiency were mild and it was not always necessary to improve them, we decided to see the patients without steroid supplementation, if adrenal insufficiency did not manifest. Therefore, we did not confirm the definitive diagnosis of adrenal insufficiency based on the clinical improvement by steroid supplementation in all patients. After this study, three patients (cases 2, 15, and 16) among the seven patients diagnosed with adrenal insufficiency suffered from symptoms, signs, and laboratory test results, such as appetite loss and hypoglycemia, when they had some infectious diseases. We administered hydrocortisone, and symptoms, signs, and laboratory test results disappeared promptly. These facts indicate that our diagnosis of adrenal insufficiency was correct. In the near future, we would like to perform a continuous study to confirm adrenal insufficiency by steroid supplementation and compare the levels of serum cholinesterase and plasma ferritin before and after 
steroid supplementation. Finally, intravenous iron formulation after HD session once a week or oral phosphate binder including iron was administered to two and one patient, respectively. It is possible that the drug administration influenced the results of iron status. However, these were patients without adrenal insufficiency. Therefore, the administration did not influence the relationships between serum cortisol levels and plasma ferritin levels in patients with adrenal insufficiency. In addition, ESA utilizes serum iron in the process of erythropoiesis. Therefore, it is possible that ESA usage influenced iron status in this study. However, no significant differences were found about present ESA usage between the patients with and without adrenal insufficiency.

\section{Conclusions}

This study indicated that it is difficult to infer adrenal insufficiency in HD patients by subjective symptoms, signs, and images of adrenal glands, and that serum cortisol level $8.45 \mu \mathrm{g} / \mathrm{dL}$ had the highest sensitivity and specificity to diagnose adrenal insufficiency in HD patients. In addition, this study indicated that adrenal insufficiency correlated with nutritional and inflammatory status, and levels of serum cholinesterase and plasma ferritin might be useful markers to predict adrenal insufficiency in HD patients.

\section{Abbreviations}

HD: Hemodialysis; BP: Blood pressure; CRP: C-reactive protein; $\mathrm{CRH}$ : Corticotropin-releasing hormone; ACTH: Adrenocorticotropic hormone; BIA: Bioimpedance analysis; nPCR: Normalized protein catabolic rate; BMl: Body mass index; ROC: Receiver operating characteristic;

ESA: Erythropoiesis-stimulating agent

\section{Supplementary Information}

The online version contains supplementary material available at https://doi. org/10.1186/s41100-021-00337-9.

Additional file 1:. Supplementary Table 1: Characteristics of the patients suspected to have adrenal insufficiency.

\section{Acknowledgements}

The authors acknowledge Taro Aoki, Daiki Goto, Takeshi Tashiro, Soichiro Nagata, Takashi Matsuyama, and Yoshitaka Naito (Internal Medicine 1; Division of Nephrology, Hamamatsu University School of Medicine) for working as primary physicians during admission and performing hormone stimulation tests. Moreover, the authors acknowledge Keisuke Kakizawa and Miho Yamashita (Internal Medicine 2; Division of Endocrinology and Metabolism, Hamamatsu University School of Medicine) for discussing to make a final decision of adrenal insufficiency.

\section{Authors' contributions}

$\mathrm{NO}$ and YS planned the research, and all authors conducted the clinical research. NO and YS obtained written informed consent from all patients. NO wrote the first draft, and YS, SAI, and SHI reviewed the draft. NO, YS, TS, $\mathrm{SAl}, \mathrm{SHI}, \mathrm{TF}$, and HY contributed to the discussion regarding final decision on adrenal insufficiency. NO, AK, and HY wrote the final draft. All authors approved the final version of this manuscript.
Funding

There are no fundings to declare.

Availability of data and materials

Data sharing is not applicable to this article as no datasets were generated or analyzed during the current study.

\section{Declarations}

Ethics approval and consent to participate

The ethics committee of Hamamatsu University School of Medicine approved this study (No. 16-226), and we adhered to the principles of the Declaration of Helsinki. Written informed consent was obtained from all patients.

Consent for publication

Not applicable

\section{Competing interests}

The authors declare that they have no competing interests.

\section{Author details}

${ }^{1}$ Internal Medicine 1, Hamamatsu University School of Medicine, 1-20-1 Handayama Higashi-ku, Hamamatsu 431-3192, Japan. ${ }^{2}$ Hamana Clinic, 235-1 Numa, Hamakita-ku, Hamamatsu 434-0037, Japan. ${ }^{3}$ Blood Purification Unit, Hamamatsu University School of Medicine, 1-20-1 Handayama Higashi-ku, Hamamatsu 431-3192, Japan.

Received: 6 December 2020 Accepted: 29 March 2021

Published online: 09 April 2021

\section{References}

1. Auron M, Raissouni N. Adrenal insufficiency. Pediatr Rev. 2015;36(3):92-102. https://doi.org/10.1542/pir.36-3-92.

2. Bancos I, Hahner S, Tomlinson J, Arlt W. Diagnosis and management of adrenal insufficiency. Lancet Diabetes Endocrinol. 2015;3(3):216-26. https:// doi.org/10.1016/S2213-8587(14)70142-1.

3. Suzuki R, Morita $H$, Nishiwaki $H$, Yoshimura A. Adrenal insufficiency in a haemodialysis patient. NDT Plus. 2010;3(1):99-100. https://doi.org/10.1093/ ndtplus/sfp143.

4. Sakao Y, Sugiura T, Tsuji T, Ohashi N, Yasuda H, Fujigaki Y, et al. Clinical manifestation of hypercalcemia caused by adrenal insufficiency in hemodialysis patients: a case-series study. Intern Med. 2014;53(14):1485-90. https://doi.org/10.2169/internalmedicine.53.1104.

5. Barton CH, Vaziri ND, Gordon S, Eltorai I. Endocrine pathology in spinal cord injured patients on maintenance dialysis. Paraplegia. 1984;22(1):7-16. https://doi.org/10.1038/sc.1984.4.

6. Mazanec K, McClure J, Bartley CJ, Newbould MJ, Ackrill P. Systemic amyloidosis of $\beta 2$ microglobulin type. J Clin Pathol. 1992;45(9):832-3. https://doi.org/10.1136/jcp.45.9.832.

7. Yanase T, Tajima T, Katabami T, Iwasaki Y, Tanahashi Y, Sugawara A, et al. Diagnosis and treatment of adrenal insufficiency including adrenal crisis: a Japan Endocrine Society clinical practice guideline [Opinion]. Endocr J. 2016; 63(9):765-84. https://doi.org/10.1507/endocrj.EJ16-0242.

8. Loutradis C, Sarafidis PA, Ferro CJ, Zoccali C. Volume overload in hemodialysis: diagnosis, cardiovascular consequences, and management. Nephrol Dial Transplant. 2020:gfaa182. https://doi.org/10.1093/ndt/gfaa182.

9. Shinzato T, Nakai S, Fujita Y, Takai I, Morita H, Nakane K, et al. Determination of Kt $\mathrm{N}$ and protein catabolic rate using pre- and postdialysis blood urea nitrogen concentrations. Nephron. 1994;67(3):280-90. https://doi.org/10.11 59/000187980.

10. Martin-Grace J, Dineen R, Sherlock M, Thompson CJ. Adrenal insufficiency: physiology, clinical presentation and diagnostic challenges. Clin Chim Acta. 2020;505:78-91. https://doi.org/10.1016/j.cca.2020.01.029.

11. Bossola M, Di Stasio E, Monteburini T, Parodi E, Ippoliti F, Bonomini M, et al. Intensity, duration, and frequency of post-dialysis fatigue in patients on chronic haemodialysis. J Ren Care. 2020;46(2):115-23. https://doi.org/1 0.1111 /jorc. 12315

12. Kuipers J, Oosterhuis JK, Krijnen WP, Dasselaar JJ, Gaillard CAJM, Westerhuis $R$, et al. Prevalence of intradialytic hypotension, clinical symptoms and nursing interventions--a three-months, prospective study of 3818 
haemodialysis sessions. BMC Nephrol. 2016;17(1):21. https://doi.org/10.1186/ s12882-016-0231-9.

13. Bodner G, Peer G, Zakuth V, Spirer ZH, Aviram A. Dialysis-induced eosinophilia. Nephron. 1982;32(1):63-6. https://doi.org/10.1159/000182805.

14. Kang DH, Lee Y, Kleine CE, Lee YK, Park C, Hsiung JT, et al. Eosinophil count and mortality risk in incident hemodialysis patients. Nephrol Dial Transplant. 2020;35(6):1032-42. https://doi.org/10.1093/ndt/gfz296.

15. Gosmanov AR, Gosmanova EO, Kovesdy CP. Evaluation and management of diabetic and non-diabetic hypoglycemia in end-stage renal disease. Nephrol Dial Transplant. 2016;31(1):8-15. https://doi.org/10.1093/ndt/gfv258.

16. Straub RH, Cutolo M. Glucocorticoids and chronic inflammation. Rheumatology (Oxford). 2016;5(Suppl 2):ii6-iii4.

17. Maraj M, Kuśnierz-Cabala B, Dumnicka P, Gala-Błądzińska A, Gawlik K, Pawlica-Gosiewska D, et al. Malnutrition, inflammation, atherosclerosis syndrome (MIA) and diet recommendations among end-stage renal disease patients treated with maintenance hemodialysis. Nutrients. 2018;10(1):69. https://doi.org/10.3390/nu10010069

18. Ichikawa H, Sugimoto M, Sakao Y, Sahara S, Ohashi N, Sano K, et al. Eradication therapy for Helicobacter pylori infection improves nutrition status in Japanese hemodialysis patients: a pilot study. J Clin Biochem Nutr. 2019;64(1):91-5. https://doi.org/10.3164/jcbn.18-61.

19. Yamamoto M, Saito H, Uejima C, Tanio A, Tada Y, Matsunaga T, et al. Combination of serum albumin and cholinesterase levels as prognostic indicator in patients with colorectal cancer. Anticancer Res. 2019;39(2):108590. https://doi.org/10.21873/anticanres.13217.

20. Sato T, Yamauchi H, Suzuki S, Yoshihisa A, Yamaki T, Sugimoto K, et al. Serum cholinesterase is an important prognostic factor in chronic heart failure. Heart Vessels. 2015;30(2):204-10. https://doi.org/10.1007/s00380-0140469-8.

21. Nemeth E, Valore EV, Territo M, Schiller G, Lichtenstein A, Ganz T. Hepcidin, a putative mediator of anemia of inflammation, is a type II acute-phase protein. Blood. 2003;101:2461-3.

22. Black S, Kushner I, Samols D. C-reactive protein. J Biol Chem. 2004;279(47): 48487-90. https://doi.org/10.1074/jbc.R400025200.

\section{Publisher's Note}

Springer Nature remains neutral with regard to jurisdictional claims in published maps and institutional affiliations.

Ready to submit your research? Choose BMC and benefit from:

- fast, convenient online submission

- thorough peer review by experienced researchers in your field

- rapid publication on acceptance

- support for research data, including large and complex data types

- gold Open Access which fosters wider collaboration and increased citations

- maximum visibility for your research: over $100 \mathrm{M}$ website views per year

At $\mathrm{BMC}$, research is always in progress.

Learn more biomedcentral.com/submissions 